\section{ANEMIA Y FISURAS LABIOPALATINAS}

\section{ANEMIA AND CLEFTS OF THE LIP AND PALATE}

\author{
Marcos Roberto Tovani-Palone ${ }^{1, a}$
}

Sr. Editor. En vista de la alta prevalencia de fisuras labiopalatinas a nivel mundial, que se estima en alrededor de 1:700 nacidos vivos, de su etiología multifactorial y de las diversas alteraciones asociadas ${ }^{(1-}$ 4), se destaca la existencia de alteraciones nutricionales (presentes luego del nacimiento) en los niños con estos defectos congénitos, ya sea por las dificultades que experimentan las madres a la hora de dar de lactar o por el miedo generado por las alteraciones anatómicas y funcionales presentes ${ }^{(3)}$.

Por otra parte, teniendo en cuenta los resultados del estudio de Pajuelo et al. ${ }^{(5)}$, que refieren una alta tasa de prevalencia de anemia, especialmente en niños de 0 11 meses de edad, de bajo peso al nacer, pobres y con madres adolescentes sin ningún nivel de instrucción, se establece un cuadro situacional de falla en la atención primaria, teniendo en consideración que se tratan de niños con condiciones morfológicas dentro de los rangos normales y con mayores posibilidades de realizar la lactancia materna.

Estos resultados(5) ${ }^{(1)}$ evan a la reflexión, ya que además de las dificultades nutricionales, las cirugías para rehabilitación de las fisuras labiopalatinas que son realizadas tempranamente, como por ejemplo en el Hospital de Reabilitação de Anomalias Craniofaciais da Universidade de São Paulo (HRAC/USP) donde a partir de los tres meses de edad se operan para la corrección de defectos de labio. Estas cirugías requieren de un peso mínimo adecuado para cada paciente, con la finalidad de obtener por lo menos un margen mínimo de seguridad de las condiciones de salud asociadas con la anestesia general ${ }^{(3)}$.

Sin embargo, no se encuentran en la literatura estudios sobre la prevalencia de la anemia y sus variables en la población de niños con fisuras labiopalatinas, por lo que se presenta la oportunidad de generar investigaciones que podrían beneficiar a las instituciones que ofrecen

\footnotetext{
1 Hospital de Rehabilitación de Anomalías Craneofaciales de la Universidad de São Paulo. Bauru, Brasil.

a Especialista en Odontopediatría, máster en ciencias de la rehabilitación Recibido: 17-08-15 Aprobado: 02-09-15
}

esta modalidad de tratamiento, además de promover estrategias para la promoción de la salud, con mejores chances de cumplir con los protocolos adoptados, reduciendo considerablemente el tiempo y el costo de todo el tratamiento.

Fuente de financiamiento: autofinanciado.

Conflictos de interés: el autor declara no tener conflictos de interés en la publicación de este artículo.

\section{REFERENCIAS BIBLIOGRÁFICAS}

1. Saldias-Vargas VP, Tovani-Palone MR, Moura-Martins AP, da Silva-Dalben G, Ribeiro-Gomide M. Enamel defects in permanent first molars and incisors in individuals with cleft lip and/or palate. Rev Fac Med. 2014;62(4):515-9. doi: http:// dx.doi.org/10.15446/revfacmed.v62n4.45598.

2. Tovani-Palone MR, Saldias-Vargas VP, Ribeiro da Silva T. Viabilidade na prescrição de antibióticos para crianças com fissura labiopalatina durante o tratamento odontológico. Rev Fac Med. 2015;63(2):331-3. doi: http://dx.doi. org/10.15446/revfacmed.v63n2.48624.

3. Tovani-Palone MR. Fissuras labiopalatinas, ganho de peso e cirurgias: leite materno versus fórmulas lácteas. Rev Fac Med. (Próximo) (pedir al autor el código DOI) 2015;63(4):695-8 doi:http://dx.doi.org/10.15446/revfacmed.v63.n4.49226.

4. Tovani-Palone MR. Fonoaudiologia e fissuras labiopalatinas. Rev Fac Med. 2015;63(4):741-2 doi:http://dx.doi. org/10.15446/revfacmed.v63.n4.51710

5. Pajuelo J, Miranda M, Zamora R. Prevalencia de deficiencia de vitamina a y anemia en niños menores de cinco años de Perú. Rev Peru Med. Exp. Salud Publica. 2015;32(2):245-51. doi: http://dx.doi.org/10.17843/rpmesp.2015.322.1614

Correspondencia: Marcos Roberto Tovani Palone

Dirección: Rua Silvio Marchione, 3-20, Vila Universitária CEP 17012-900, Bauru, SP, Brasil.

Teléfono: +55 (14) 3235-8141/ Fax +55 (14) 3234-7818

Correo electrónico:marcos_palone@hotmail.com

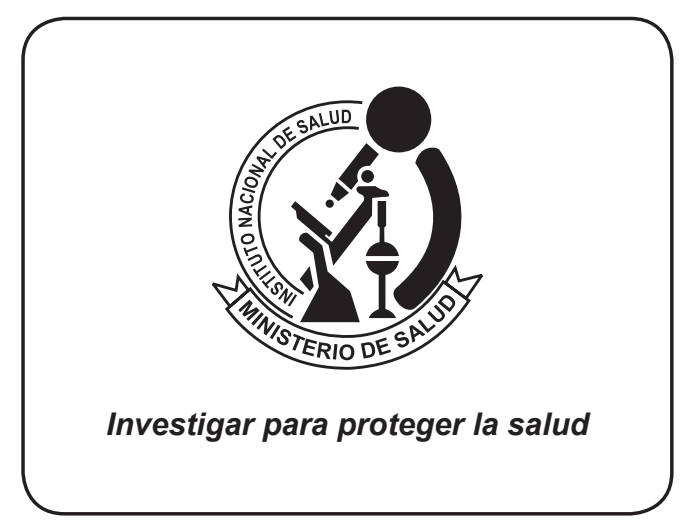

